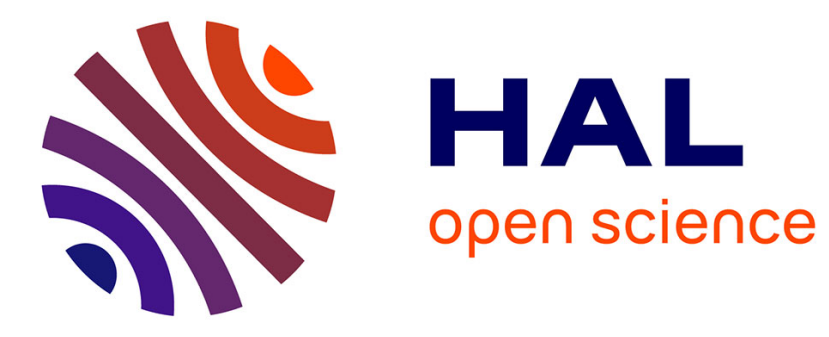

\title{
L'obsession de la vocation chez les musicien(ne)s underground
}

\author{
Jean-Marie Seca
}

\section{To cite this version:}

Jean-Marie Seca. L'obsession de la vocation chez les musicien(ne)s underground. Revue internationale de psychosociologie et de gestion des comportements organisationnels, 2002, VIII (18), pp. 177-188. 10.3917/rips.018.0177 . hal-03006263

\section{HAL Id: hal-03006263 \\ https://hal.univ-lorraine.fr/hal-03006263}

Submitted on 17 Nov 2020

HAL is a multi-disciplinary open access archive for the deposit and dissemination of scientific research documents, whether they are published or not. The documents may come from teaching and research institutions in France or abroad, or from public or private research centers.
L'archive ouverte pluridisciplinaire $\mathbf{H A L}$, est destinée au dépôt et à la diffusion de documents scientifiques de niveau recherche, publiés ou non, émanant des établissements d'enseignement et de recherche français ou étrangers, des laboratoires publics ou privés.

\section{(이) $\$$}

Distributed under a Creative Commons Attribution - NonCommercial - NoDerivatives $\mid 4.0$ 


\title{
Résumé
}

L’approche psychosociologique des pratiques musicales populaires alternatives, ici présentée, synthétise une partie des résultats d'une enquête approfondie de terrain (par les méthodes du questionnaire, de l'entretien et de l'observation participante) auprès de 110 groupes (quatre cent dix individus appartenant aux courants pop rock, punk, new-wave, rap, musique antillaise), effectuée en 1981-87 et 2000, dont les énoncés complets figurent dans d'autres publications (Seca, 2001a). Après avoir décrit le modèle théorique (état acide) à partir duquel ont été traité les données d'observation et une fois indiqué quelle est la spécificité de l'approche de ce type de musicien, on se penche plus particulièrement sur la question de la place des femmes dans ces pratiques, très majoritairement masculines. On part du présupposé que les conflits de rôles féminins / masculins émergent de façon plus explicite dans la manière qu'ont ces dernières de s'afficher ou de revendiquer une "place» dans le champ social de reconnaissance musicale. Quelques pistes d'interprétation de l'ostracisme rencontré par les musiciennes et de leurs comportements provocateurs et paradoxaux sont proposées. La structure de rôle androgynique (valable pour les deux sexes dans le cas de l'activité artistique) est invoquée comme un point d'achèvement d'un parcours de professionnalisation artistique et comme une « solution » aux excès et aux dérives identitaires possibles dans la recherche créative musicale underground.

\begin{abstract}
The psychosociological approach of the alternative popular musical practices, here presented, synthetize a part of the results of detailed empirical inquiry (by the methods of the questionnaire, the interview and the participating observation) with 110 groups (approximately four hundred and ten individuals) belonging to the pop currents (rock, punk, new-wave, rap and caribbean styles), carried out in 1981-87 and 2000, whose complete statements appear in others publications (Seca, 2001a). After having described the theoretical model (acid state) from which were treated the data of observation and once indicated which is the specificity of the approach of this type of musician, one bends more particularly over the question of the place of the women in these practices, very mainly masculinized. We are leaving from the presupposition that the conflicts of feminine / male roles appear in a more explicit way in the manner which have these last ones to display or to claim a "place" in the social field of musical recognition. Some tracks of the interpretation of the ostracism, met by the female musicians and of their provocative and paradoxical behaviors, are proposed. The androgynic structure of role (valid for the two sexes in the case of artistic activity) is called like a process of completion in the artistic professionnalisation and as a " solution " to the excesses and to the possible drifts of the identity in the underground musical creative research.
\end{abstract}



psychosociologie, vol. VIII, n 18, numéro spécial « Autour de l’Art et des Arts », pp. 177-188.

\section{Problématisation}

Scène de vie festive dans un quartier de Paris, réputé populaire et « mixte socialement » autant qu'ethniquement, en septembre 2001 : l'événement a lieu dans un square récemment réaménagé, pas très loin de la ZUP de La Grange aux Belles. Sur les tréteaux, des rappers convaincus scandent leur texte, en l'entrecoupant par des «Ouais ! Ouais ! Ouais ! », rythmés et phatiques. Ils sont très jeunes : neuf, dix ou onze ans pour la plupart. D’autres groupes, composés de membres plus âgés, leur succèdent. Des revendications s'enchaînent et s'articulent aux rimes. Les formations donnent leur spectacle sous le patronage d'une maison de quartier et de la mairie d'arrondissement. L'underground bien neuf, vraiment jeune, tout frais sorti des classes primaires et de sixième, est là devant nos yeux : les fils et les filles d'Afrique, d'Europe, d’Amérique et du Maghreb, réunis dans un hip hop de bon aloi, révolté et groove réveillent l' « esprit de La Chapelle » qui était un terrain vague, situé près du métro du $10^{\mathrm{e}}$ arrondissement, ayant constitué l'une des premiers regroupement rap underground de la Capitale, au milieu des années quatre-vingt. Au pied de la scène, des auditeurs prépubères et d'autres, adolescents, apprécient et encouragent leurs pairs qui se risquent face au regard d'autrui. Tout autour, des badauds, parfois attentifs, souvent indifférents, continuent leur promenade du dimanche. Il est 18 heures. Soudain, un groupe de jeunes filles de dix / quinze ans apparaît. Elles sont toutes habillées de la même manière. Surprise ! Enfin, du nouveau dans le ciel rap et rock? Voyons ce qu'elles vont dire et comment elles vont s’y prendre ! Contrairement à leurs camarades garçons, rappers et musiciens, elles se mettent à danser sur un thème musicalement connu. Elles préfèrent donc s’exhiber dans une représentation chorégraphique bien réglée et orchestrée.

À chacun selon son statut de sexe ? Aux garçons, la revendication et les performances provocatrices argotisées, «mentalisées » et poétisées ? Aux filles les manifestations plus harmonieuses, communicatives et pacifiques de la rythmique corporelle et de la danse ? L’observation inopinée de ces traductions différenciées de la " cause » rap, qu’on croirait directement prélevées et « remixées » à partir d’observations sur les permanences structurales des « inégalités » hommes / femmes dans les tâches domestiques, illustre l'existence de modalités spécifiques de réception et d'appropriation du même style musical. En plus de constituer des discours rebelles convenus, des ensembles révoltés, commercialement conformes, les styles underground (rock, rap, techno) se transmuent en pratiques presque majoritaires, " acceptées », encouragées, plébiscitées et même promues au rang de techniques éducatives de rue. Elles finissent par devenir, sous le sceau et l'approbation des divers décideurs politiques, pédagogiques, socioculturels, des moyens de diffusion de représentations et d’activités conformistes, par moments populistes et parfois thérapeutiquement rageuses. On peut désormais parler de l'existence d'un underground de masse et de sa généralisation comme mode de vie et de pensée.

La réflexion sur l'incidence du statut de sexe dans ces pratiques sera, dans cet article, un révélateur pour l'analyse des tendances et des représentations inhérentes à ces courants. Dans cette contribution, le caractère artistique de ces styles musicaux ne sera ni vanté, ni critiqué, ni encore moins approfondi. Après avoir présenté le modèle théorique qui nous a servi d'outil d'analyse de ces milieux, nous tenterons de comprendre comment les «filles jouent aux musiciennes » et si les conflits de rôles qui en résultent, versent, pour reprendre un langage connoté comme « actuel », dans la complaisance "phallocentriste », machiste ou normative ou bien, au contraire, si la manière dont elles s’impliquent dans de telles activités est fort proche de celle des « garçons » et n'est susceptible de recevoir aucune attribution stigmatisante, conformiste et sexuée.

Le corpus d'étude sur lequel nous prenons appui, consiste en entretiens, en questionnaires et en observations participantes (auprès de 2 groupes entièrement féminins de punk rock, de 22 groupes mixtes -hommes / femmes- et de 87 groupes entièrement masculins) ainsi que dans l’observation de sites web consacrés à des figures féminines de l'underground dont la fameuse Louise Madonna Ciccone, célèbre star américaine, spécialiste patentée de la provocation, d'entreprises financièrement très rentables et du chant soul et rock (Trédez, 2000 ; Schwichtenberg, 1993). Vu la faiblesse de nos effectifs empiriques féminins, notre approche peut être qualifiée de « clinique des pratiques rock telles que perçues par des femmes ». Quelques travaux sur les musiques populaires selon le sexe peuvent être consultés dans les ouvrages en anglais de Frith (1990) ou de Whitheley (1997). En France, le livre d’Escal et Rousseau-Dujardin (1999) est centré plutôt sur le cas des compositrices du genre « classique ».

\section{Représentations musicales et état acide}

Rappelons quel est notre point de vue psychosociologique sur les musicien(ne)s pop rock, rap, techno et leurs pratiques. Les genres musicaux, quels qu'ils soient, sont assimilables à des reconstructions culturelles viables, échangeables et vendables sur un "marché symbolique ». Ils structurent les objets et les transactions esthétiques entre un nombre plus ou moins grand de suiveurs - imitateurs et un ensemble indéterminé d’innovateurs - artistes. Ce sont des 

psychosociologie, vol. VIII, n 18, numéro spécial « Autour de l’Art et des Arts », pp. 177-188.

représentations sociales $(=R S)$ et plus particulièrement «musicales ». Les $R S$ forment, comme on le sait, des classes de savoirs pratiques, de comportements, d'opinions, de croyances, de sentiments et de valeurs plus ou moins fortement associés aux combinaisons artistiques et figuratives. Sur le plan musical, elles sont des systèmes d'ambiance, de sentiments, de formes esthétiques réactivées lors de rituels plus ou moins figés ou rénovés (Moscovici, 1976 ; Seca, 2001b). Le tissage d'un lien entre les $R S$ et leurs effets comportementaux est, dans le cas des musiques populaires à visée innovante, plus remarquable et plus affirmé que dans d'autres champs sociaux ou culturels. On peut percevoir, dans l'activité musicale, une dimension rituelle majeure. En effet, celle-ci suppose « un système codifié de pratiques, sous certaines conditions de lieu et de temps, ayant un sens vécu et une valeur symbolique pour ses acteurs et ses témoins, en impliquant la mise en jeu du corps et un certain rapport au sacré » (Maisonneuve, 1999, p. 12.) Comme le souligne Durkheim, " les rites sont des manières d'agir qui ne prennent sens qu'au sein des groupes assemblés et qui sont destinés à susciter, à entretenir ou à refaire certains états mentaux de ces groupes. » (Durkheim, 1912, p. 13). Nos observations auprès de 110 ensembles de musique amateurs ou underground (punk, rock, antillais, rap) de 1982 à 2000 , nous ont amené à construire une grille d'analyse, qualifiée d' "état acide ", par laquelle nous décrivons le mode de socialisation, de représentation et d'atteinte de buts éthiques et économiques de reconnaissance publique de ces formations.

Plus précisément, l'état acide est défini comme la formalisation théorique d'une forme d'errance psychologique, d'une situation exacerbée, émotionnellement et cognitivement parlant, de transition professionnelle et de recherche d'orientation dans un contexte anomique et de déviance. Il renvoie donc à l'état mental et social, à la fois actif et mélancolique, de minorités qui se cherchent un code et un rituel conformes à ce qu'elles considèrent être leur « nature ». De ce point de vue, ce modèle est applicable à d'autres types d'activités, analogues du point de vue professionnel, politique ou identitaire (Seca, 1998). On décrira ses principales dimensions, ci-après, en ne considérant que les pratiques musicales pop.

La première caractéristique à mettre en avant dans ce mode d'approche des musiciens underground est celle de l'ambivalence. Ces derniers sont agités, tourmentés par une forme de mimétisme dénégateur. En effet, contrairement aux pratiques folkloriques ou populaires traditionnelles, ces praticiens cherchent à affirmer une singularité personnelle en dépit d'une identification très nette à des courants, styles ou vedettes adorés. L’ambivalence qui en résulte naît d'une tension entre deux « soi » : la première entité est constituée par la figure du participant à une foule enthousiaste qu’a été, ou est encore, tout membre d'un groupe amateur ; la seconde tendance, plus «identitaire » et personnelle, cristallise une vocation et une intériorité psychologique privée / publique, à faire émerger dans les combinatoires musicales, poétiques ou unaires (Dufour, 1989). L'état acide et l'ambivalence sont alors engendrés par la friction et les ajustements entre deux types d'identités: l'une étant fusionnelle, fraternisante, admirative et soumise ; l'autre se destinant à l'individualisation, à la dissidence, à l'assomption d'un leadership charismatique et à la construction d'une culture « autolégitimante », éprise de liberté et d’originalité. Des drames et des dilemmes se jouent et se résolvent dans les deux espaces. Il s'agit, soit de se départir d'une tendance conformiste (fusion bienfaisante dans la masse admirative, striée, sculptée au moyen de signes totémiques communs autovalorisants), soit de jouer le va-tout de la particularité quasi snob, parfois fondée sur une recherche spirituelle personnelle, avec les risques de demeurer un « inconnu amer » ou un « fou de soi », victime hystérique de visions impalpables et de révoltes avortées.

À partir de cet attribut essentiel (ambivalence et mimétisme dénégateur), on peut ensuite passer aux différents traits de ces pratiques néo-rituelles et de l'état acide :

2. Expression d'une volonté d’influencer les médias, les conventions régissant les communications et les institutions, voire de transformer leur idéologie latente ;

3. Désir relatif de changer la situation d'anonymat et de faible visibilité commerciale à son propre profit ;

4. Dépendance forte vis-à-vis du style des vedettes, des modèles culturels en cours et des pressions du show business ;

5. Vocation pour la mission de « leader de foule » et pour la conversion d'autrui à sa production privée et à la vision du monde qu'elle implique ;

6. Survalorisation des moments d'effervescence et d'intensité lors des concerts et des célébrations qui leur sont associées ;

7. Implication dans des rituels semi-privés de répétition et de construction d’une identité virtuelle, partiellement concrétisée dans un répertoire et des «morceaux » enregistrés sur cassettes ou disques ou dans des regroupements ou des coopérations ponctuelles avec divers partenaires ; 

psychosociologie, vol. VIII, n 18, numéro spécial « Autour de l’Art et des Arts », pp. 177-188.

8. Représentation de la musique comme « pure », fondée sur les notions d'authenticité, de son grunge, dépouillé, bruitiste ;

9. Esthétique de la provocation, teintée d'arrière-pensée pécuniaires, et diffusion par différents moyens de la production groupale (featuring, réseaux associatifs, médias traditionnels, disques, internet...).

Les acteurs dont nous parlons ici sont minoritaires. Ils envisagent une carrière après avoir été des consommateurs passionnés de styles pop rock. Ils sont, aussi, anomiques du fait de leur ambivalence / attirance (plus ou moins déniée ou moquée) vis-à-vis des stratégies de réussite et des honneurs (argent, admiration, renommée) qu'elle suppose. Ils sont dépendants du regard des majorités parce qu'ils n’ont pas encore constitué leur style propre et leur devise musicale (cf. Rouget 1980 ; Seca, 2001a). N’ayant pas suffisamment affirmé et matérialisé ostensiblement leur propre originalité, ils sont tiraillés entre des visées symboliques et des valeurs peu conciliables : la réalisation de soi, le goût pour le travail bien fait, l'authenticité, la rébellion « jeune », le sexe, l'argent, la vie facile ou le scandale dont on connaît la nature dynamique, mais aussi la charge utopique et perturbatrice pour la vie sociale domestique (Moscovici, 1979). Cela explique aussi l'attirance de ces minorités musicales pour des contenus discursifs et politiques hétérodoxes : elles se constituent comme des espaces de réchauffement de la croyance dans le « soi ». Par conséquent, elles conduisent à des pratiques d'intensification de la foi dans ses propres forces, dons ou capacités, d'identification à «sa " musique, prolongeant, sur un mode subversif, l'emprise de l'idéologie individualiste.

L'état acide formalise la structure des représentations et des conduites d'acteurs expérimentant, de façon oscillante, bringuebalante, la recréation d'un style d'appréhension culturelle de leur environnement. Ces formations underground incarnent, par leurs activités et leurs productions, les non-réponses à leurs obsessions intellectuelles, affectives ou politiques, par des compromis formels « corporéisés », plus ou moins esthétiques, comme beaucoup d'autres marginaux ou provocateurs artistiques avant eux. L' " entrée dans le monde » prend alors l'apparence de mélodies et de rythmes consommés « entre soi » et de la recherche d'une combinaison rituelle commune vivable. L'esthétique est autant un écran qui s'interpose entre soi et l'extériorité sociale qu'un moyen de l’interpréter et de se la représenter.

Concernant les minorités en général, on évoquera, comme souvent dans l'analyse de problèmes génériques, un cas particulier. On pensera, en effet, aux performances étonnantes d'un rapper (dont on respectera l'anonymat), qui est bègue "dans la vie de tous les jours ", incapable de "parler normalement" ou même, bien sûr, de produire une argumentation lors de transactions ordinaires, sans buter sur son handicap verbal. Or, durant ses raps, il ne commet pas un seul accident de parcours, "déchirant » ses auditeurs par ses tournures linguistiques, énonçant ses mots et ses rimes sans hésitation, avec assurance et maîtrise. L’art du rap serait-il parfois thérapeutique ? Il se présente, en tout cas, comme un des langages du corps et de l'esprit minoritaire. Le bégaiement de cet individu et sa temporaire dilution dans l'activité rap ne sont-ils pas les signes de son rapport passionné à la transe, au plaisir de la parole et à la communauté retrouvée ? Dany-Robert Dufour, dans son livre Le bégaiement des maîtres, relève d'ailleurs les effets sémantiques et philosophiques de certaines «perles redondantes » jusque dans les discours des fondateurs du structuralisme dans les sciences humaines contemporaines (Dufour, 1989).

Ces diverses caractéristiques, émergentes, revendicatives, identitaires et souffrantes, attribuées usuellement aux cultures minoritaires peuvent aussi être accolées, "mariées" aux pratiques expressives et culturelles des artistes féminins. Si on revient sur l'état acide et sa structure, il n’y a nul doute que les femmes correspondent à un groupe social sensible à l'aigreur du temps, susceptible de prêter l'oreille à la hargne ambivalente et oscillante du rock. Ne s'agit-il pas d'une population en situation parfois anomique, souvent contestataire et fréquemment déviante ? Certaines jeunes filles, qu'on peut observer dans quelques quartiers de Paris, se saluent d'ailleurs à la manière des rappers, en se frappant le poing puis en claquant le paume de leurs mains d'un coup bref et apaisant. Rien ne différencie, en apparence, la socialisation des deux sexes dans ce domaine comme dans d'autres : même ouverture à la consommation de masse, aux fringues, à la vie épicurienne et aux modes. Et pourtant, les choses sont plus complexes et subtiles. Les féministes patentées ne sont pas souvent des amatrices de hip hop (du moins pas toujours) et les fabricants de rap féminins ou les danseuses de hip hop ne se posent pas fréquemment et explicitement des questions féministes ; du moins, elles ne les affinent pas en les insérant dans une approche doctrinale. Les mêmes oppositions plus ou moins hybrides (féministes artistes, artistes féminins «machistes », ou refusant toute discussion de ces problèmes) existent d'ailleurs dans d'autres arts.

\section{Y a-t-il un mode d'appropriation « féminin » de l'underground ?}

L'expression d'un blues ou d'un spleen associé au vécu oppressif émanant du contexte de vie féminin (sujétion professionnelle, sociale, domestique, lutte pour l'affirmation de soi, conflit latent, hégémonie des modèles traditionnels 

psychosociologie, vol. VIII, n 18, numéro spécial « Autour de l’Art et des Arts », pp. 177-188.

de sexe, idéologies ou utopies gay, queer...) ne devrait qu’y favoriser le développement croissant, et même le pullulement, de pratiques créatives rap, rock ou techno. Or, d’après nos enquêtes et nos observations dans les milieux musicaux de ce type, il n’en est rien. Les «minorités femmes » sont encore plus minoritaires dans les réseaux d’art libertaire et de pratique alternative. Jusqu’ici du moins, les musiques underground sont essentiellement accaparées et produites par des sujets masculins (neuf individus sur dix lors d'une des rares enquêtes quantitatives dans ces milieux ; Seca, 2001a ; voir aussi d'autres études : Bayton, 1997 ; Cohen, 1997), même si des madones ou des princesses de la musique hip hop, soul, house ou rock surgissent et disparaissent sporadiquement sur les chaînes de télévisions thématiques (MTV par exemple) diffusant des vidéo-clips. L’affirmation d’une vie underground féminine semble difficile à avancer. Elle mérite ainsi d’être interrogée, au-delà du fait statistique lui-même, pour tenter de cerner quels sont les rites adoptés et les représentations diffusées par ce type d’artisanes, parfois courtisanes ou ensorceleuses. Leurs conduites sont certes modelées par une pensée libertaire, subversive, mais aussi par une vision masculine du groupe et de l'individuation.

On sait que certains mouvements lesbiens se sont associés, au moins temporairement, à des courants pop (Mannisch Girl, Riot Grrrl, tendances queer). Le mode de cette appropriation est souvent ostentatoire. On revendique une aptitude à la performance artistique à "égalité supérieure » de dérive avec les hommes. Peut-on parler d'une spécificité féministe, explicitement destinée à bousculer les « conventions », des messages produits par les groupes féminins ? La parodie est l'une des réponses courantes dans ce domaine, surtout lorsque l’on veut subvertir les clivages dans les représentations du genre. La "féminisation » ostentatoire et accentuée de certaines lesbiennes (ports de vêtements classiquement féminins comme les robes bouffantes, les jupes courtes, les tailleurs distingués mais aussi les lingeries vulgaires, les objets fétichistes, les maquillages, etc.) peut être contrebalancée, à l'opposé, par une «masculinisation » du comportement encore plus banalement contestataire. La provocation sexuelle de quelques vedettes féminines est tout autant une stratégie commerçante qu'une une manière d'afficher une «identité insaisissable " en résonance avec l'image que certains hommes en ont. Ce qui remarquable dans ces « fantasmes » répétitifs et diffus, c'est la fascination « jeuniste », voire celle d'un « lolitisme » à la Nabokov. En effet, l'ère et l'esprit " jeune » ont pris avec la culture des teen-agers, l'apparence troublante de la féminité légère, « juste » pubère, tour à tour délicate et vulgaire, orientée vers le culte de la vitesse et le rythme haletant du rock.

Avec les musiques issues du blues ou du jazz, électrifiées, bruitistes et très cadencées, l'affichage d'une forme débridée d'usage du corps et du plaisir, associé à une certaine exhibition, se diffuse de façon explicite. La question, débattue dans divers écrits, est de savoir si ce type d'art provocateur, lascivement corporéiste, appartient ou non au pôle masculin, majoritaire ou encore « hétéro-normatif », pour reprendre une expression glanée dans la littérature récente sur les mouvements gays ou lesbiens (Bourcier, 2001). D’autres voix plus prosaïques dénoncent une commercialisation fructueuse de l'érotisme sous une forme volontiers «fétichiste » (Winter, 2001). Il semble que nous soyons dans un paysage brouillé où l'affirmation branchée du sexe voisine avec les images les plus éculées du genre. Même si la position critique (utiliser les clichés pour les détourner et les faire exploser), souvent adoptée par divers artistes, est plausible, ceux-ci ne sont pas à l'abri d’une complaisance envers ce qu'ils feignent de persifler. De plus, l'ambiguïté fondamentale des arts rock et underground dont nous avons essayé de dégager la spécificité « acide ", les accule à la trivialité (adhérer aux stéréotypes sous prétexte de se vouloir populaire) ou au snobisme ampoulé (se prétendre aussi sophistiqué que les « grands » ou les « génies des arts »). Une troisième option apparaît : il s’agit de la mauvaise estime de soi qu'ont eu nombre de musiciens plus ou moins innovateurs et underground. Pour comprendre cela, il suffit de visionner la scène d'un film, réalisé en 1988 par Clint Eastwood, Bird, consacré au génial Charlie Parker, où ce denier se retrouve, titubant, défoncé, sortant d'une voiture, devant la barrière de l'entrée de la maison d’Igor Stravinsky dont il guette l'éventuelle apparition et à qui il voue un culte. Concernant les musiciennes, ce raisonnement est tenable. Les torsions que certaines font subir à des images vulgaires ou à des conduites malséantes qu'elles font mine d'endosser cyniquement, ne les désengagent pas d'une participation minimale à l'acte dénié. Leur dépendance larvée à des stéréotypes qu'elles prétendent combattre, les minent; ce qui est pourtant compréhensible lorsqu'on poursuit une revendication identitaire. Par nature, toute identité est arrachée à une autre dont on veut s'affranchir ; une peau recouvre l'autre sans faire disparaître les couleurs et la forme de l'ancienne.

Mais en toute occurrence, la compétition entre femmes et hommes demeure, avec un risque d'exclusion des musiciennes de certains milieux underground. Les diverses enquêtes dans ces environnements (Bayton, op. cit. ; Cohen, op. cit. ; Seca, op. cit.) indiquent que les « jouets » des jeunes postadolescents sont jalousement gardés par ces derniers. Les jeunes filles qui tentent d'accéder au terrain de football, au manche de la guitare électrique et à la caisse claire de la batterie, ne font-elles pas irruption dans un territoire réservé ? Et il doit y avoir chez nombre de ces musiciennes une vieille rancœur, macérée dans des souvenirs de cour de récréation ou de quelque envie envers certains garçons de la famille à qui l'on réserve tendanciellement certains rôles. L'histoire de vie des chanteuses célèbres d'Edith Piaf à Janis 

psychosociologie, vol. VIII, n 18, numéro spécial « Autour de l’Art et des Arts », pp. 177-188.

Joplin, de Louise Madonna Ciccione à Courtney Love, illustre cette mémoire de la domination latente des « hommes » que l'on tente d'inverser ou de faire « mentir » par une consécration professionnelle atypique. Finalement, c'est bien sur ce terrain de la légitimation par l'œuvre accomplie que maints tourments identitaires débouchent ; on peut parler d'une forme de résolution des problèmes personnels par leur mise en scène dans le champ des carrières artistiques. La vogue des reality shows et de situations plus ou moins perverses où des groupes d' « anonymes " sont brusquement mis en vedette, comme Loft story et Star Academy des chaînes de télévision privées, démontre à quel degré la diffusion de l'intime à l'échelle du grand nombre devient un met de consommation courante et une éthique de remplacement pour les «aventuriers du coin de la rue ». Ce narcissisme «publicisé » est fort bien évoqué chez les jeunes musiciennes suivantes :

«- e2 (bassiste) : c'est quelque chose qui vient de toi...

- e3 (batteuse) : de toi et que tu essaies de transmettre...

- e2 (bassiste) : quand tu crées quelque chose, cela vient de toi... et quand tu es sur ta basse ou ta batterie, ça doit se sortir! Ça doit se voir. Les gens doivent sentir que c'est toi. Que c'est toi qui joue! » (Trafic Diams, groupe de punk rock, Paris, 1985)

"e1(guitariste) : L'esprit du groupe c'est nous! Oui, je crois que c'est vraiment nous. On n'a pris aucune idée extérieure en essayant de coller dessus, se disant: "on va faire ça parce qu'on aime bien ça." Non! C'est vraiment nous ! » (Les Aristochattes, groupe de punk rock, Paris, 1983).

Un entretien, effectué en 1999, avec une jeune pianiste, éduquée dans une famille de musiciens classiques professionnels, ayant refusé de rentrer dans le moule du Conservatoire et des cours de piano académiques illustre le même type de discours. Cette compositrice de trip hop énonce son engagement sur le même mode : appropriation hypersensible d'une forme esthétique estimée plus conforme à une «nature» qui serait «au fond de soi » et personnalisation d'une musique et de son style dont l'énonciatrice sait bien qu'ils ont une provenance collective. À cet égard, on devrait supposer que les jeunes filles, comme d'autres groupes minoritaires, devraient se diriger vers les arts et la communication beaucoup plus fréquemment que les garçons. Cette orientation vers des territoires où l'on peut débattre de soi en s'exprimant dans la création concerne d'ailleurs aussi le champ des activités scientifiques (Lage 1993 ; Mariotti, 2001). Si le combat féministe a un sens, ne doit-on pas le voir réémerger naturellement et fréquemment dans l'affirmation d'un engagement et d'un épanouissement professionnels ?

« - e3 (batteuse) : on s'est dit "pourquoi pas nous ?" On n’a pas calculé le coup tu vois.

- e1 (guitariste) : et puis après, en comparaison, on s'est dit : "on a quand même un impact. On est un groupe de nanas !" On s'en est rendu compte très tard. Il y a un an et demi de ça. Tout le monde venait: "Oh un groupe de filles ! Mais c'est le zoo ici !" Il y avait des gens qui venaient dans notre local et qui regardaient. On avait l'impression d'être en cage $[. .$.$] .$

- E : D’un seul coup, au bout de trois mois de répétitions, vous vous êtes aperçues que vous étiez un groupe de filles?

- e1 (guitariste) : ben oui !

- e3 (batteuse) : on ne savait pas jouer! Et on s'est dit "pourquoi pas nous ?" » (Trafic Diams, groupe punk rock, Paris, 1985)

«e1 (guitariste) : À l'époque il y avait le Rose Bonbon et les musiciens entraient gratuitement là-bas. Nous, on en avait marre de payer [rire] et un jour on a vu qu'il y avait un hommage à John Lennon. Ils prenaient n'importe quel groupe sans audition. Alors pour nous, cela a été le point de départ. Et puis, on a continué. En fait, on aimait bien quoi! Je ne sais pas. On n'avait pas envie de rester dans l'ombre, dans une cave, toute une vie. On ne joue pas pour rester au fond d'une cave mais pour être sur une scène et voir des gens. » (Les Aristochattes, groupe punk rock, Paris, 1983)

Finalement, rien ne différencie la rhétorique féminine de la motivation de celle des groupes masculins dans ce domaine. Les $R S$ du travail, du plaisir esthétique, de la réussite, de l'engagement pour voir, des influences revendiquées, la recherche d'une visibilité sociale, l'obsession du "pourquoi pas nous ?", de ce que nous nommons le mimétisme dénégateur et, plus généralement, ce que nous qualifions d' " état acide », caractérisent largement les premiers pas de bien des groupes depuis des décennies. L'importance accordée au fluide musical, à une conception onaniste ou narcissique de la création («Ça vient de toi ! " «C'est ce que tu as de plus sincère en toi ») ou les préoccupations matérielles («la tune »), techniques ou instrumentales sont narrées de façon similaire. Ce qui surprend le plus c’est surtout la manière dont on se réfère au statut sexuel des interviewées dans les divers extraits. Les musiciennes 

psychosociologie, vol. VIII, n 18, numéro spécial « Autour de l’Art et des Arts », pp. 177-188.

interrogées feignent de s'être engagées dans un groupe entièrement féminin sans l'avoir fait vraiment exprès, sans volontarisme, ni provocation, ni encore moins pour des raisons obscures de marketing. Si l'on cherche la coloration féminine du discours, peut-être doit-on le chercher dans l'affirmation d'une égalité de don et de créativité et dans l'invocation d'une amitié communautaire.

« Guitariste : On trouvait ça marrant d'être un groupe de filles. Et puis, on a peut-être les mêmes délires avec des filles. » (Les Aristochattes, groupe punk rock, Paris, 1983)

Ce type d'allusion à l'appartenance fusionnelle n’est pas spécifiquement féminin, bien que les travaux de Lorenzi-Cioldi sur les groupes "agrégats» ou "collection» laissent supposer et observer une tendance plus « communautariste », à la présentation massive et « massée » du soi chez les femmes (Lorenzi-Cioldi, 1988). Au cours de nos enquêtes, la représentation agrégative et indifférenciée affleure en permanence chez tous les musiciens amateurs ou underground, qu'ils soient de sexe masculin ou féminin, en tendant ainsi vers une forme psychosociale d'androgynie. Celle-ci ne serait-elle pas un mode d' «être au monde» spécifique aux créateurs ? L'androgynie psychologique (Lorenzi-Cioldi, 1994) influence-t-elle, de façon partielle, le développement d'une appropriation des styles musicaux alternatifs? Apparaît-elle comme un ensemble d'arrivée, c'est-à-dire comme le résultat d'une progression vers "autre chose " chez un musicien ou une musicienne ? Résulte-t-elle d'un investissement dans la création plus qu'elle ne détermine certains individus à se sortir des schémas conventionnels propre à chaque sexe ? À ce stade de notre réflexion, on ne peut que noter l'effet structurant et, même, vitalisant du mouvement d'affirmation et de construction du soi quasi androgynique dans le processus d'accès à l'autonomie artistique et créative.

\section{Bibliographie}

Bayton M., 1997, « Women and the Electric Guitar », in Witheley S. (Éd.), Sexing..., op. cit., pp. 37-49.

Bourcier, M.-H., 2001, Queer zones. Politiques des identités sexuelles, des représentations et des savoirs, Paris, Balland.

Cohen, S., 1997, « Men Making a Scene. Rock and the Production of Gender », in Witheley S. (Éd.), Sexing..., op. cit., pp. 17-36.

Dufour, D.-R., 1989, Le bégaiement des maîtres, Paris, François Bourin.

Durkheim, E., 1912, Les formes élémentaires de la vie religieuse, Paris, PUF (4e éd. : 1960).

Escal, F. et Rousseau-Dujardin, J., 1999, Musique et différence des sexes, Paris, L'Harmattan.

Frith, S. et Mac Robbie, A, 1990, «Rock and sexuality », in Frith, S. et Goodwin, A. (Éd.), On Record : Rock, Pop and the Written Word, New York, Pantheon Book, pp. 371-389.

Lage, E., 1993, Lycéens et pratiques scientifiques. Comment les sciences deviennent une passion, Paris, L’Harmattan.

Lorenzi-Cioldi F., 1988, Individus dominants et groupes dominés. Images masculines et féminines, Grenoble, PUG.

Lorenzi-Ciodi F., 1994, Les androgynes, Paris, PUF.

Maisonneuve, J., 1999, Les conduites rituelles, Paris, PUF ( $3^{e}$ édition corrigée).

Mariotti, F., 2000, Études expérimentales des représentations sociales de la science et des métiers scientifiques selon le sexe au collège et au lycée, Thèse pour le doctorat de psychologie sociale, Saint-Denis, Université Paris-8.

Moscovici, S., 1976, La psychanalyse, son image, son public, (nouv. éd. augmentée), Paris, PUF (1 $1^{\mathrm{re}}$ édition : 1961).

Moscovici, S., 1979, Hommes domestiques et hommes sauvages, Paris, Christian Bourgeois.

Rouget, G., 1980, La musique et la transe. Esquisse d'une théorie générale de la musique et de la possession, Paris, Gallimard.

Schwichtenberg, C. (Éd.), 1993, The Madonna Connection : Representational Politics, Subcultural Identities, and Cultural Theory, St. Leonards, NSW, Allen and Unwin..

Seca, J.-M., 1998, « Pour une psychologie sociale de la vocation minoritaire » (trad. en roumain : « Pentru o psihologie socialã a vocatiei minoritari »), in Neculau A. et Ferréol G. (Éd.), Psihosociologia schimbãrii, Iasi, Polirom, pp. 68-76.

Seca, J.-M., 2001a, Les musiciens underground, Paris, PUF.

Seca, J.-M., 2001b, Les représentations sociales, Paris, A. Colin.

Trédez, F., 2000, Madonna, Paris, Librio.

Winter, J.-P., 2001, Les errants de la chair. Etudes sur l'hystérie masculine, Paris, Payot (1 $1^{\text {re }}$ édition : 1998).

Witheley S. (Éd.), 1997, Sexing the Groove. Popular Music and Gender, London, Routledge. 